International Journal on Cybernetics \& Informatics ( IJCI) Vol.3, No.1, February 2014

\title{
Global Stabilization of a Class of Nonlinear System Based on Reduced-order State Feedback Control
}

\author{
Chang-Zhong Chen ${ }^{1,2,}$ Tao Fan ${ }^{1,2}$, Bang-Rong Wang ${ }^{3}$, Dong-Ming Xie ${ }^{1,2}$ and \\ Ping $\mathrm{He}^{1,2}$ \\ ${ }^{1}$ School of Automation and Electronic Information, Sichuan University of Science \& \\ Engineering, Zigong, Sichuan 643000, People's Republic of China; \\ ${ }^{2}$ Artificial Intelligence Key Laboratory of Sichuan Province, Sichuan University of \\ Science \& Engineering, Zigong, Sichuan 643000, People's Republic of China; \\ ${ }^{3}$ Department of Mathematics, School of Science, Sichuan University of Science \& \\ Engineering, Zigong, Sichuan 643000, People's Republic of China.
}

\begin{abstract}
The problem of global stabilization for a class of nonlinear system is considered in this paper.The sufficient condition of the global stabilization of this class of system is obtained by deducing thestabilization of itself from the stabilization of its subsystems. This paper will come up with a designmethod of state feedback control law to make this class of nonlinear system stable, and indicate the efficiency of the conclusion of this paper via a series of examples and simulations at the end. Theresults presented in this paper improve and generalize the corresponding results of recent works.
\end{abstract}

\section{KEYWORDS}

Nonlinear systems; Reduced-order control; Global stabilization.

\section{Introduction}

Stabilization is a seriously important topic in control system designing [1]-[13]. In recent years the stabilization of nonlinear systems has won extensive attention from researchers, and some achievements has been made. The problem is simplified into the question of stabilization of low order systems, the sufficient condition of the system is gained, and that the state feedback control law is designed by combining centre manifold theory with part feedback linearization (e.g., [14]).

The design method to construct global stabilization feedback control law is founded and several sufficient condition of global stabilization for the system is gained by applying linearization method to a class of nonlinear systems (e.g., $[15,16])$. The sufficient condition of local stabilization and global stabilization for the system is gained by researching the stabilization question of minimum phase nonlinear system (e.g., [16]). A new method to design control laws is given, and it has been proved that the corresponding close-loop system can be globally stable under appropriate condition by researching the stabilization question of a class of affine nonlinear

DOI: $10.5121 /$ ijci.2014.3102 
system with standard form (e.g., [17]). The sufficient condition for the global stabilization of several smooth feedback is given by researching the global stabilization of cascade systems constructed with linear systems which can be made stable and asymptotically stable nonlinear systems (e.g., [18]). The stabilizationquestion of a class of triangle system is researched with smooth output feedback (e.g., [19]). The global stabilization question of cascade system made up of two nonlinear systems is researched via constructing Lyapunov function (e.g., [20]). The sufficient condition for the system to be stable is gained, and a state feedback control law is designed out, through researching the stabilization question of a class of non-minimum phasenonlinear system with its reduced-order control system(e.g., [21]). The sufficient condition for the system to be stable is gained, and that a state feedback control law is designed out through through researching the stabilization question of a class of non-minimum phase-nonlinear system based on drive control (e.g., [22]).

This paper will research the global stabilization question of a class of nonlinear system on the base of analysis, (e.g. See Ref. [8]-[12]), and deduce the stabilization of the original system from the stabilization of its subsystem-reduced-order control system. According to the features of this class of nonlinear system, this paper will come up with a design method for the corresponding state feedback control law, and will prove that close-loop system is globally asymptotically stable under appropriate condition with Lyapunov second method. This paper will indicate the efficiency of the conclusion of this paper via a series of examples and simulations at the end. In this paper, if not specially illustrated, $\mathrm{k} \cdot \mathrm{k}$ refers to Euclid norm, $\mathrm{k} \cdot \mathrm{kF}$ refers to Frobenius norm, $|\cdot|$ refers to scalar function or absolute value of function.

\section{System analysis}

Consider the following nonlinear system

$$
\left\{\begin{array}{l}
\dot{x}=f(x)+2 h(x, y) y \\
\dot{y}=u+g(x, y) y
\end{array}\right.
$$

where $\mathrm{x} \in \mathrm{Rm}$ and $\mathrm{y} \in \mathrm{Rn}-\mathrm{m}$ are state vectors, $\mathrm{u} \in \mathrm{Rn}-\mathrm{m}$ is a input vector. $\mathrm{f}(\mathrm{x})$ is a vector

function of $\mathrm{x}, \mathrm{h}(\mathrm{x}, \mathrm{y})$ is a vector function of $(\mathrm{x}, \mathrm{y}), \mathrm{g}(\mathrm{x}, \mathrm{y})$ is a scalar function of $(\mathrm{x}, \mathrm{y})$, and $\mathrm{f}(\mathrm{x})$, $\mathrm{g}(\mathrm{x}, \mathrm{y})$ and $\mathrm{h}(\mathrm{x}, \mathrm{y})$ are at least $\mathrm{C} 1$ functions about $\mathrm{x}$ or $(\mathrm{x}, \mathrm{y})$, respectively.

Assumption 1. The subsystem of system (1)

$$
\dot{x}=f(x) \text {. }
$$

is globally stable.

Remark 1. This paper uses the static stability of system (1) to discuss its dynamic stability. The purpose of Assumption 1 is to ensure the static stability of system (1).

In order to obtain main result of this paper, we should do further supposition.

Assumption 2. The subsystem (2) of system (1) is globally stable and there is a Lyapunov 
function $\mathrm{v}(\mathrm{x})$ which satisfies

$$
\begin{aligned}
\left.\dot{v}(x)\right|_{(2)} & =\frac{\partial v(x)}{\partial x} f(x) \\
& =\left[\frac{\partial v(x)}{\partial x_{1}} \frac{\partial v(x)}{\partial x_{2}} \cdots \frac{\partial v(x)}{\partial x_{m}}\right]\left[\begin{array}{l}
f_{1}(x) \\
f_{2}(x) \\
\vdots \\
f_{m}(x)
\end{array}\right] \\
& <-1, \forall\|x\| \geq M .
\end{aligned}
$$

where $\mathrm{M}$ is a positive constant.

Before proving main theorems, present the following analysis and Lemma 1 at first. Consider the following nonlinear system

$$
\left\{\begin{array}{l}
\dot{x}=f(x, y), \\
\dot{y}=-y g(x, y) .
\end{array}\right.
$$

where $\mathrm{x} \in \mathrm{Rm}$ and $\mathrm{y} \in \mathrm{Rn}-\mathrm{m}$ are state vectors, $\mathrm{f}(\mathrm{x}, \mathrm{y})$ and $\mathrm{g}(\mathrm{x}, \mathrm{y})$ are vector function of $(\mathrm{x}, \mathrm{y})$, and $\mathrm{f}(\mathrm{x}, \mathrm{y})$ and $\mathrm{g}(\mathrm{x}, \mathrm{y})$ are at least $\mathrm{C} 1$ functions about(x, y).

Lemma 1. (e.g. see Ref. [23]) If the subsystem of system (3)

$$
\dot{x}=f(x, 0) \text {. }
$$

is globally stable, and there is a continuous differentiable scalar functions $\mathrm{v}(\mathrm{x})$ such that limkxk $\rightarrow 0 \mathrm{v}(\mathrm{x})=+\infty$, and the derivative of along the trajectory of system (4) at k x k>0 such that $\mathrm{v}(\mathrm{x}) \leq 0$, then system (3) is globally stable.

Remark 2. Lemma 1 obtained is rather significant for the proving of the main theorems in this paper, the main idea of which is that the stabilization of the original system can be proved when reduced-order subsystem such that static stabilization and some essential assumed conditions. Before the theorem of this paper given, we must give the following Lemma 2 at first, which is is easy to understand.

Lemma 2. (e.g. see Ref. [26], [27]) Assume that subsystem (2) of nonlinear system (1) is globally asymptotically stable, then from Converse-Lyapunov theorem, we know that there must be a positive function $\mathrm{v}(\mathrm{x})$ which satisfies

$$
\begin{aligned}
\left.\dot{v}(x)\right|_{(2)} & =\frac{\partial v(x)}{\partial x} f(x) \\
& =\left[\frac{\partial v(x)}{\partial x_{1}} \frac{\partial v(x)}{\partial x_{2}} \cdots \frac{\partial v(x)}{\partial x_{m}}\right]\left[f_{1}(x), f_{2}(x), \cdots, f_{m}(x)\right]^{T} \\
& \leq 0, \forall x \in R^{m}-\{0,0, \cdots, 0\} .
\end{aligned}
$$


Without loss of generality. We can suppose

$$
\dot{v}(x) \leq-\varepsilon<0, \forall x \in R^{m}-\{0,0, \cdots, 0\} .
$$

where " is a very small positive.

\section{Main results}

In the process of researching nonlinear systems, it's always desired that the states of the system can reach stable values, so that as many interferences as possible could be avoided. Therefore, making systems stable has a strong practical applicability. Because of that this question has a certain actual application background, the research in this paper has some practical meaning.

\subsection{Reduced-order state feedback controller design}

Theorem 1. Assume that subsystem (2) of nonlinear system (1) satisfies Assumption 2 and there is a scalar function $\mathrm{K}(\mathrm{x}, \mathrm{y})$ for system (1) which satisfies:

$$
K(x, y)>\left\|\left[\frac{\partial v(x)}{\partial x_{1}} \frac{\partial v(x)}{\partial x_{2}} \cdots \frac{\partial v(x)}{\partial x_{m}}\right]\right\|_{2}^{2} \cdot\|h(x, y)\|_{F}^{2} .
$$

Then there is a state feedback control low

$$
\left[\begin{array}{c}
u_{1} \\
u_{2} \\
\vdots \\
u_{n-m}
\end{array}\right]=\left[\begin{array}{c}
-y_{1} K(x, y) \\
-y_{2} K(x, y) \\
\vdots \\
-y_{n-m} K(x, y)
\end{array}\right]-\left[\begin{array}{c}
y_{1} g(x, y) \\
y_{2} g(x, y) \\
\vdots \\
y_{n-m} g(x, y)
\end{array}\right]
$$

such that the system (1) globally asymptotically stable, where the elements of $K(x, y)$ are at least $\mathrm{C}_{1}$ functions about $(\mathrm{x}, \mathrm{y})$.

Proof. After substitution the state feedback control low (7) into system (1) we see that

$$
\left\{\begin{array}{l}
\dot{x}=f(x)+2 h(x, y) y \\
\dot{y}=-y K(x, y)
\end{array}\right.
$$

According to Lemma 2, we can construct a Lyapunov function

$$
V(x, y)=v(x)+\frac{1}{2} y^{T} y .
$$


then its derivative along the trajectory of system (1) is

$$
\begin{aligned}
\left.\dot{V}(x, y)\right|_{(1)}= & {\left[\frac{\partial v(x)}{\partial x_{1}} \frac{\partial v(x)}{\partial x_{2}} \cdots \frac{\partial v(x)}{\partial x_{m}}\right]\left[\begin{array}{c}
f_{1}(x) \\
f_{2}(x) \\
\vdots \\
f_{m}(x)
\end{array}\right] } \\
& +2\left[\frac{\partial v(x)}{\partial x_{1}} \frac{\partial v(x)}{\partial x_{2}} \cdots \frac{\partial v(x)}{\partial x_{m}}\right] \cdot h(x, y) y+\|y\|^{2} \cdot[-K(x, y)] \\
\leq & 0+\left\|\left[\frac{\partial v(x)}{\partial x_{1}} \frac{\partial v(x)}{\partial x_{2}} \cdots \frac{\partial v(x)}{\partial x_{m}}\right]\right\| \cdot\|h(x, y)\|_{F} \cdot\|y\|+\|y\|^{2} \cdot[-K(x, y)] .
\end{aligned}
$$

Combined with condition (6) we easy to know

$$
\dot{V}(x)<0, \forall x \in R^{m}-\{0,0, \cdots, 0\} .
$$

Then we can conclude from Lemma 1 and analysis, e.g. see Ref. 8-12, that system (1) is globally asymptotically stable.

\subsection{Further result in mathematics}

The condition of subsystem (2) respect to system (1) is weakens and the further investigation is given.

Theorem 2. Assume that subsystem (2) of nonlinear system (1) is satisfies Assumption 1. Then there must be a positive $M$, when $K(x, y)+g(x, y)<-M$, there is a state feedback control low

$$
\left[\begin{array}{c}
u_{1} \\
u_{2} \\
\vdots \\
u_{n-m}
\end{array}\right]=\left[\begin{array}{c}
y_{1} K(x, y) \\
y_{2} K(x, y) \\
\vdots \\
y_{n-m} K(x, y)
\end{array}\right]
$$

such that the system (1) globally asymptotically stable, where the elements of $K(x, y)$ are at least $\mathrm{C}_{1}$ functions about $(\mathrm{x}, \mathrm{y})$.

Proof. After substitution the state feedback control low (9) into system (1) we see that

$$
\left\{\begin{array}{l}
\dot{x}=f(x)+2 h(x, y) y, \\
\dot{y}=y K(x, y)+g(x, y) y
\end{array}\right.
$$


According to Lemma 2, we can construct a Lyapunov function

$$
V(x, y)=v(x)+\frac{1}{2} y^{T} y
$$

then its derivative along the trajectory of system (1) is

$$
\begin{aligned}
\left.\dot{V}(x, y)\right|_{(1)}= & {\left[\frac{\partial v(x)}{\partial x_{1}} \frac{\partial v(x)}{\partial x_{2}} \cdots \frac{\partial v(x)}{\partial x_{m}}\right]\left[\begin{array}{c}
f_{1}(x) \\
f_{2}(x) \\
\vdots \\
f_{m}(x)
\end{array}\right] } \\
& -\varepsilon+2\left[\frac{\partial v(x)}{\partial x_{1}} \frac{\partial v(x)}{\partial x_{2}} \cdots \frac{\partial v(x)}{\partial x_{m}}\right] \cdot h(x, y) y+\|y\|^{2} \cdot[K(x, y)+g(x, y)] \\
\leq & 0+\left\|\left[\frac{\partial v(x)}{\partial x_{1}} \frac{\partial v(x)}{\partial x_{2}} \cdots \frac{\partial v(x)}{\partial x_{m}}\right]\right\| \cdot\|h(x, y)\|_{F} \cdot\|y\| \\
& +\|y\|^{2} \cdot[K(x, y)+g(x, y)] .
\end{aligned}
$$

Combined with condition $K(x, y)+g(x, y)<-M$ we easy to know that so long as

$$
M>\frac{\left\|\left[\frac{\partial v(x)}{\partial x_{1}} \frac{\partial v(x)}{\partial x_{2}} \cdots \frac{\partial v(x)}{\partial x_{m}}\right]\right\|_{2}^{2} \cdot\|h(x, y)\|_{F}^{2}}{\varepsilon}
$$

there must be

$$
\dot{V}(x)<0, \forall x \in R^{m}-\{0,0, \cdots, 0\}
$$

Then we can conclude from Lemma 1 and analysis, e.g. see Ref. 8-12, that system (1) is globally asymptotically stable.

\section{System simulations}

In this section, in order to show that the approach of this paper to this sore of control system is effective and convenient, we give some illustrative examples and simulation results.

Example 1. Consider the following second-order nonlinear system

$$
\left\{\begin{array}{l}
\dot{x}=-x^{3}+2 y^{2} \\
\dot{y}=u+3 x y^{2}+y^{2}
\end{array}\right.
$$


Solution. Base on the theorem above, for the subsystem of system (11), we can construct a Lyapunov function which is given by

$$
v(x)=x^{2}
$$

It is easy to know that the subsystem of system (11)

$$
\dot{x}=-x^{3} \text {. }
$$

is globally asymptotically stable.

Base on the condition (6) of Theorem 1, let

$$
K=4 x^{2} y^{2} .
$$

Then, there is a state feedback control law

$$
u=-4 x^{2} y^{3}-3 x y^{2}-y^{2} .
$$

such that the system (11) is globally asymptotically stabilization.

Simulation. Without loss of generality. Let the initial values of simulation

$$
\left\{\begin{array}{l}
x(0)=-4 \\
y(0)=+3
\end{array}\right.
$$

and the input control signal

$$
u= \begin{cases}0.5, & \text { for } t \geq 0 \\ 0, & \text { for } t<0\end{cases}
$$

The sample time is $0.1 \mathrm{~s}$, and the simulation time are $50 \mathrm{~s}$ seconds.

The dynamic response of the system (11) with the state feedback control law (12) is show in Figure 1. 
From the Figure 1, we can know that the controlled closed-loop system of the system (11) is asymptotically stable.

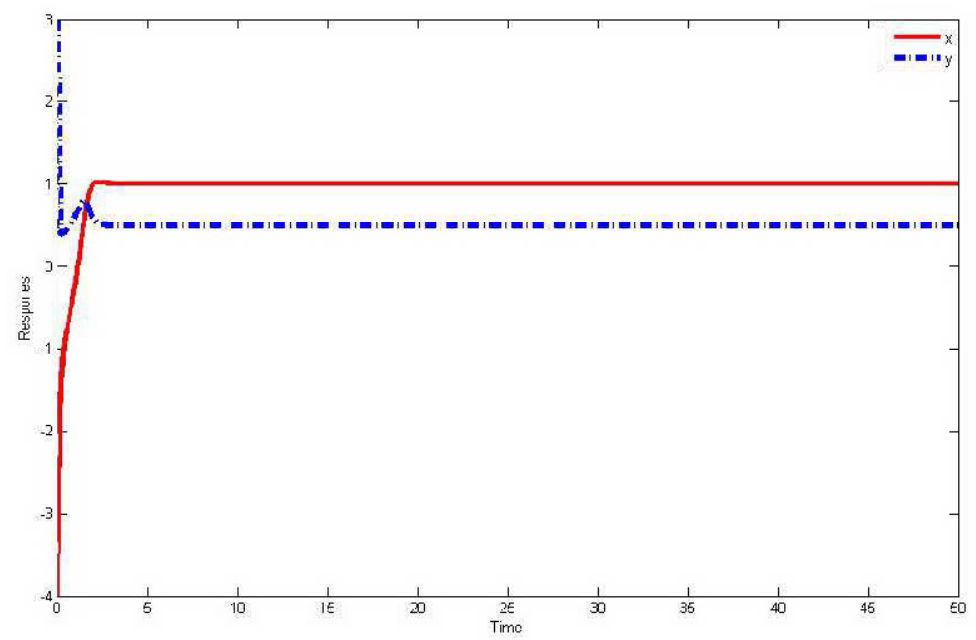

Figure 1. Dynamic response of the system (11) with the state feedback control law (12).

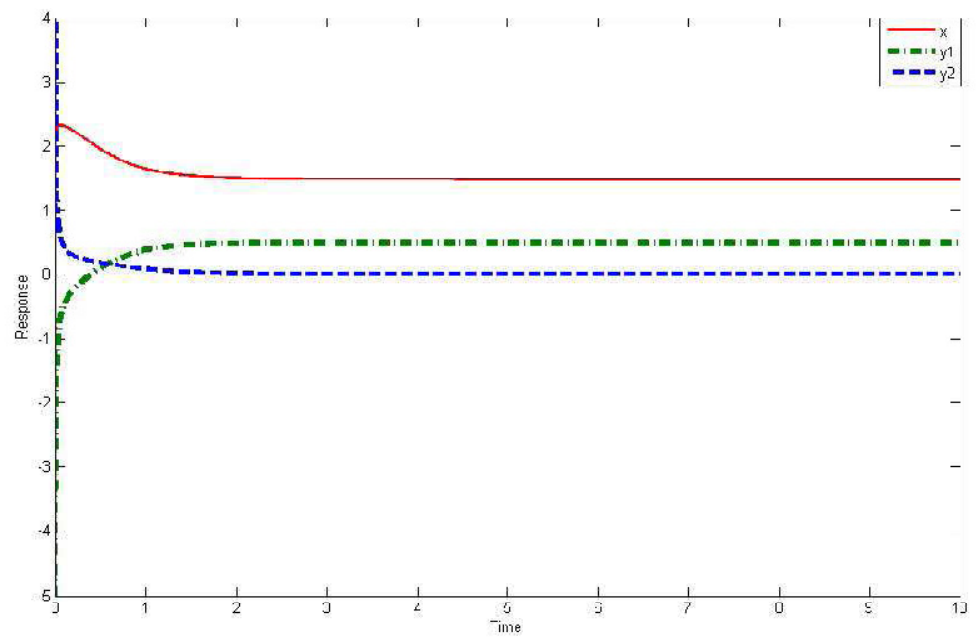

Figure 2. Dynamic response of the system (13) with the state feedback control law (14).

Example 2. Consider the following third-order nonlinear system

$$
\left\{\begin{array}{l}
\dot{x}=-x^{3}+2 y^{2} \\
\dot{y}_{1}=u_{1}+x y_{1}^{2}+x y_{1} y_{2}+x y_{1} y_{2}^{3} \\
\dot{y}_{2}=u_{2}+x y_{1} y_{2}+x y_{2}^{2}+x y_{2}^{4}
\end{array}\right.
$$


Solution: Base on the theorem above, for the subsystem of system (13), we can construct a Lyapunov function which is given by

$$
v(x)=\frac{1}{2} x^{2} .
$$

It is easy to know that the subsystem of system (13)

$$
\dot{x}=-x \text {. }
$$

is globally asymptotically stable.

Base on the condition (6) of Theorem 1, let

$$
K=x^{2}\left(2 y_{1}^{2}+2 y_{2}^{2}\right)+1 \text {. }
$$

Then, there is a state feedback control law

$$
\left\{\begin{array}{l}
u_{1}=-2 x^{2} y_{1}^{3}-2 x^{2} y_{1} y_{2}^{2}-y_{1}-x y_{1}^{2}-x y_{1} y_{2}-x y_{1} y_{2}^{3} \\
u_{2}=-2 x^{2} y_{1}^{2} y_{2}-2 x^{2} y_{2}^{2}-y_{2}-x y_{1} y_{2}-x y_{2}^{2}-x y_{2}^{4}
\end{array}\right.
$$

such that the system (13) is globally asymptotically stabilization.

Simulation. Without loss of generality. Let the initial values of simulation

$$
\left\{\begin{array}{l}
x(0)=2 \\
y_{1}(0)=-5 \\
y_{2}(0)=4
\end{array}\right.
$$

and the input control signal

$$
u= \begin{cases}1, & \text { for } t \geq 0 \\ 0, & \text { for } t<0\end{cases}
$$

The sample time is $0.1 \mathrm{~s}$, and the simulation time are $10 \mathrm{~s}$ seconds.

The dynamic response of the system (13) with the state feedback control law (14) is show in Figure 2.

From the Figure 2, we can know that the controlled closed-loop system of the system (13) is asymptotically stable [28]. 
International Journal on Cybernetics \& Informatics ( IJCI) Vol.3, No.1, February 2014

\section{Conclusion}

Based on some work of pioneers, the global stabilization problem for a class of nonlinear system is investigated in this paper, and that the stabilization of the original system is obtioned from the stabilization of its reduced-order subsystem. A design method for the corresponding state feedback control law is given according to the features of this class of nonlinear system, and that the closeloop system is globally asymptotically stable under appropriate condition is proved with Lyapunov second method. This paper has indicated the efficiency of the conclusion of this paper via a series of examples and simulations at the end.

\section{Acknowledgment}

The authors would like to express their sincere appreciation to Professor Gong-Quan Tan and Xiao-Hong Ren for some valuable suggestions toward achieving the work of this paper. The authors wish to thank the editor and reviewers for their conscientious reading of this paper and their numerous comments for improvement which were extremely useful and helpful in modifying the manuscript. This work was jointly supported by the Open Foundation of Enterprise Informatization and Internet of Things Key Laboratory of Sichuan Province(Grant No. 2013WYY06), the Open Foundation of Artificial Intelligence Key Laboratory of Sichuan Province (Grant Nos. 2014RYY02, 2013RYJ01, 2012RYJ01 and 2011RYY05), the Science Foundation of Sichuan University of Science \& Engineering (Grant No. 2012KY19) and the Research Foundation Department of Education of Sichuan Province (Grant No. 11ZA121).

\section{References}

[1] P.He, S. H. Ma, and T. Fan, "Finite-time mixed outer synchronization of complex networks with coupling time-varying delay," Chaos, vol. 22, no. 4, pp. 043151, Dec. 2012.

[2 H.Niu, S. H. Ma, T. Fan, C. Chen, P. He. "Linear state feedback stabilization of unified hyperchaotic systems," World Journal of Modelling and Simulation, vol. 10, no. 1, pp. 34-48, Feb.

2014.

[3] P.He, Q. Zhang, C. Jing, C. Chen, T. Fan. "Robust exponential synchronization for neutral complex networks with discrete and distributed time-varying delays: A descriptor model transformation method," Optimal Control, Applications and Methods, 2013. [online] http://dx.doi.org/10.1002/oca.2094.

[4] P.He, C. Jing, T. Fan, C Chen. "Robust decentralized adaptive synchronization of general complex networks with coupling delayed and uncertainties," Complexity, 2013.[online] http://dx.doi.org/10.1002/cplx.21472.

[5] P.He, C. Jing, C. Chen, T. Fan, H. Nik. "Synchronization of general complex networks via adaptive control schemes," PRAMANA-Journal of Physics, 2014. In press.

[6] P.He, C. Jing, T. Fan, C. Chen. "Outer synchronization of complex networks with multiple coupling time-varying delays," International Journal of Control and Automation, vol. 6, no. 4, pp. 197-216, Sep. 2013.

[7] P.He, C. Jing, T. Fan, C. Chen. "Robust adaptive synchronization of complex networks with multiple coupling time-varying delays," International Journal of Automation and Control, vol.7, no. 4, pp. 223-248, Dec. 2013. 
International Journal on Cybernetics \& Informatics ( IJCI) Vol.3, No.1, February 2014

[8] P.He, and G. Q. Tan, "Hळ observer design for a class of time-delay nonlinear systems with external disturbance," International Journal of Applied Mathmatics and Mechanics, vol. 8, no. 16, pp. 67-78, June 2012.

[9] P.He, X. H. Ren, and F. H. Li, "Robust stabilization of uncertain unified chaotic systems," Journal of Central China Normal University (Natural Sciences), vol. 46, no. 1,pp. 35-39, Feb.2012.

[10] P.He, and F. Tan, "Linear state feedback stabilization for controlled chaotic systems," International Journal of Nonlinear Science, vol. 12, no. 3, pp. 373-384,Dec. 2011.

[11] C.Chen, T. Fan, B. Wang, H. Nik, P. He. 'Feedback linearization synchronization of unified chaotic systems," Journal of Applied Nonlinear Dynamics, 2014. Inpress.

[12] P.He, H. Y. Lan, and G. Q. Tan, "Delay-independent stabilization of linear systems with multiple time-delays," World Academy of Science, Engineering and Technology, vol. 51, no. 3,pp. 1007-1011, Mar. 2011.

[13] P.He, "Partial stabilization of a class of nonlinear systems via center manifold theory," World Academy of Science, Engineering and Technology, vol. 51, no. 3, pp. 1012-1024, Mar. 2011.

[14] Marino R., Feedback stabilization of single-input nonlinear system, Systems and Control Letters. 10:201-206, 1988.

[15] BaumannW., RughW., Feedback control of nonlinear systems, IEEE Transactions on Automatic Control. 31:44-47, 1986.

[16] Byres C. I., Isidori A., Asymptistic stabilization of minimum phase nonlinear systems, IEEE Transactions on Automatic Control. 36:1122-1237, 1991.

[17] Seibert P., Snarez R., Global stabilization of a certain class of nonlinear systems, Systems and Control Letters. 16:17-23, 1991.

[18] Saberi A., Kokotovic P., Suaamann H. J., Global stabilization of partially linear composite systems, SIAM Control. 28:1491-1503, 1990.

[19] Qian C., Lin W., Output feedback stabization of planar systems without controllableobservable linearization, IEEE Transactions on Automatic Control. 47:2068-2073, 2002.

[20] Mazenic F., Sepulchre R., Jankovic M., Lyapunov function for stable cascades and applications to global stabilization, IEEE Transactions on Automatic Control. 44:1795-1800, 1999.

[21] Cheng D. Z., Hu Q. X., Qin H. S., Feedback diagonal canonical form and its application to stabilization of nonlinear systems, Science in China Ser. F: Information Sciences. 48:201-210,2005.

[22] Juan C., Manuel A., Passivity based stabilization of non-minimum phase nonlinear systems,Kybernetika. 45:417-426, 2009.

[23] Outbid R., Jghima H., Comments on the stabilization of nonlinear systems by adding an integrator,IEEE Transactions on automatic control. 41:1804-1807, 1996.

[24] Cheng D. Z., Guo Y. Q., Stabilization of nonlinear systems via the center manifold approach,Systems and Control Letters. 57:511-518, 2008.

[25] LinW., Global asymptotic stabilization of general nonlinear systems with stable free dynamicsvia passivity and bounded feedback, Automatic. 32:915-924, 2009.

[26] Khalil H. K., Weber M. E., Nonlinear systems, Second etition, Prentice Hall, Inc. New Jersey,1996.

[27] Feng Y. J., Dong Y. L., Global stabilization for a class of nonlinear system, Journal of tianjinpolytechnic university. 26:69-72, 2007. (in Chinese)

[28] Fan T., Chen C. Z., Ren X. H., He P., Adaptive synchronization of delayed Chen chaotic system, Discontinuity, Nonlinearity and Complexity. 2014, in press. 
International Journal on Cybernetics \& Informatics ( IJCI) Vol.3, No.1, February 2014

\section{Authors}

Chang-Zhong Chen received the B.S. Degree in Automatic Control from Sichuan University, in Chengdu, Sichuan, People's Republic of China and the M.S. Degrees in Control Science and Engineering from Sichuan University of Science \& Engineering, in Zigong, Sichuan, China, in 1997 and 2009, respectively. He is currently an Associate Professor with the School of Automation and Electronic Information, Sichuan University of Science \& Engineering, in Zigong, Sichuan, China, from 2010. His research interests include Technology and application of microcontrollers, Intelligent control and Chaos control.

Tao Fan received the B.S. Degree and the M.S. Degree in MeasurementTechnology and Automation Device from Beijing University of Chemical Technology, in Beijing,People's Republic of China, in 2003 and 2006, respectively.He is currently a Lecturer with the School of Automation and Electronic Information, Sichuan University of Science \& Engineering, in Zigong, Sichuan, China. His main research

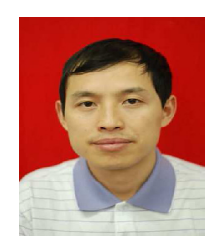
interests include Intelligent control, Chaos control and synchronization, Complex networks and Consensus of multi-agent systems. 